\begin{tabular}{|c|c|}
\hline Title & On convergence of ICCG applied to finite element equation for quasi-static fields \\
\hline Author(s) & Igarashi, H.; Honma, T. \\
\hline Citation & $\begin{array}{l}\text { IEEE Transactions on Magnetics, 38(2), 565-568 } \\
\text { https://doi.org/10.1109/20.996148 }\end{array}$ \\
\hline Issue Date & $2002-03$ \\
\hline Doc URL & http:/hdl.handle.net $/ 2115 / 38718$ \\
\hline Rights & $\begin{array}{l}\text { @ } 2002 \text { IEEE. Personal use of this material is permitted. However, permission to reprint/republish this material for } \\
\text { advertising or promotional purposes or for creating new collective works for resale or redistribution to servers or lists, } \\
\text { or to reuse any copyrighted component of this work in other works must be obtained from the IEEE. }\end{array}$ \\
\hline Type & article \\
\hline File Information & igarashi02.pdf \\
\hline
\end{tabular}

Instructions for use 


\title{
On Convergence of ICCG Applied to Finite-Element Equation for Quasi-Static Fields
}

\author{
H. Igarashi and T. Honma
}

\begin{abstract}
This paper discusses convergence of the incomplete Cholesky conjugate gradient method (ICCG) which solves edge-based finite-element equations for quasi-static electromagnetic fields. It has been observed in numerical computations that convergence of ICCG for the A-V method is faster than that for the A method. This phenomenon is found to be explained by the fact that, in the A-V method, the preconditioning eliminates the small singular values which deteriorate the condition number while they remain after the preconditioning in the case of the $A$ method.
\end{abstract}

Index Terms-Eddy current, edge element, finite-element method, ICCG, singular value.

\section{INTRODUCTION}

$\mathbf{E}$ DDY-current problems have successfully been solved by the edge-based finite-element method. There are two formulations for them: the A method [1] and the A-V method (see [2] and references therein to find the origin of the A-V method). In the A-V method, the vector potential $\boldsymbol{A}$ and scalar potential $V$ are chosen as the unknowns. In the A method, only the vector potential is chosen as the unknown variable.

Because the number of the DoFs in the A method is smaller than that in A-V method, the former seems superior to the latter. However, it has been observed that the convergence of ICCG for the A method is significantly worse than that for the A-V method, and moreover, the total computation times for the latter are often shorter than those for the former [3], [4]. The following is devoted to the analysis of these phenomena.

\section{FORMULATION}

Let us consider the quasi-static electromagnetic fields governed by

$$
\begin{aligned}
\nabla \times(\nu \nabla \times A)+\mathrm{j} \omega \sigma(\boldsymbol{A}+\nabla V) & =\boldsymbol{J}_{0} \\
\mathrm{j} \omega \nabla \cdot \sigma(\boldsymbol{A}+\nabla V) & =0
\end{aligned}
$$

where

$\nu$ magnetic reluctivity;

$\sigma$ conductivity;

$\boldsymbol{A}$ vector potential;

$V \quad$ scalar potential;

$\boldsymbol{J}_{0} \quad$ external current which satisfies $\nabla \cdot \boldsymbol{J}_{0}=\mathbf{0}$.

Note that (2) is linearly dependent on (1), since taking divergence of (1) yields (2). Also, for symmetrization of the matrix, $V$ is defined so that the relation $\boldsymbol{E}=-\mathrm{j} \omega(\boldsymbol{A}+\nabla V)$ holds.

Manuscript received June 1, 2001; revised October 25, 2001.

The authors are with the Graduate School of Engineering, Hokkaido University, Kita-ku 060-8628, Japan (e-mail: iga@em-si.eng.hokudai.ac.jp).

Publisher Item Identifier S 0018-9464(02)02422-6.
The finite-element discretization of (1) and (2) with edge elements [5] provides

$$
\left[K^{\prime}\right]\left\{\begin{array}{l}
A \\
V
\end{array}\right\}=\left\{\begin{array}{l}
J \\
0
\end{array}\right\}
$$

where

$$
\left[K^{\prime}\right]=\left[\begin{array}{cc}
{[C]^{t}[\nu][C]+\mathrm{j} \omega[\sigma]} & \mathrm{j} \omega[\sigma][G] \\
\mathrm{j} \omega[G]^{t}[\sigma] & \mathrm{j} \omega[G]^{t}[\sigma][G]
\end{array}\right]
$$

and the $f \times e$ matrix $[C]$ and $e \times n$ matrix $[G]$ are the discrete counterparts of curl and grad, whose entities consist of \pm 1 and 0 , and satisfy the relation $[C][G]=0 . n$, e, and $f$ denote the number of nodes, edges, and faces, respectively. The matrices $[\nu]$ and $[\sigma]$ are $f \times f$ positive definite and $e \times e$ positive semidefinite matrices, respectively. (We are tacitly considering the domain containing air region where $\sigma=0$.) The method in which (3) is solved is referred to as the A-V method.

The scalar potential $V$ in the above formulation can be eliminated due to the fact that grad $W^{0} \subset W^{1}[1]$, where $W^{k}$ represents the space spanned by the Whitney $k$-form. Equation (3) is then reduced to

$$
[K]\{A\}=\{J\}
$$

where

$$
[K]=[C]^{t}[\nu][C]+\mathrm{j} \omega[\sigma] .
$$

The method in which (5) is solved is called the A method.

\section{RESULTS OF A NUMERICAL EXPERIMENT}

A simple eddy-current problem [6], shown in Fig. 1, is analyzed by both the $\mathrm{A}$ and $\mathrm{A}-\mathrm{V}$ methods. In this model, ac line currents generate a time-harmonic magnetic field so that eddy currents are induced in the parallel conductors. Only 1/8 of the model is considered due to the symmetry. The tree-cotree gauging has not been applied because it is known that the gauging makes the matrix condition worse [7]. The number of iterations $N$ until the convergence of the CG process is evaluated.

Table I summarizes the dependence of $N$ on frequency, where $\alpha$ is a constant defined by $\alpha=a^{2} \omega \sigma \mu$. The constant $\alpha$ is a nondimensional quantity since it can also be expressed as $\alpha=2(a / \delta)^{2}$, where $\delta$ is the skin depth. Moreover $\kappa$ in Table I denotes the condition number of $[K]$ and $\left[K^{\prime}\right]$, which will be defined in Section IV. It can be seen that the decrease in frequency $f$ makes the convergence of $\mathrm{CG}$ worse in both the $\mathrm{A}$ and $\mathrm{A}-\mathrm{V}$ methods.

What we pay attention to is the convergence of ICCG. That is, the convergence for the A method becomes worse at smaller frequencies as in the case of the CG. On the other hand, the 


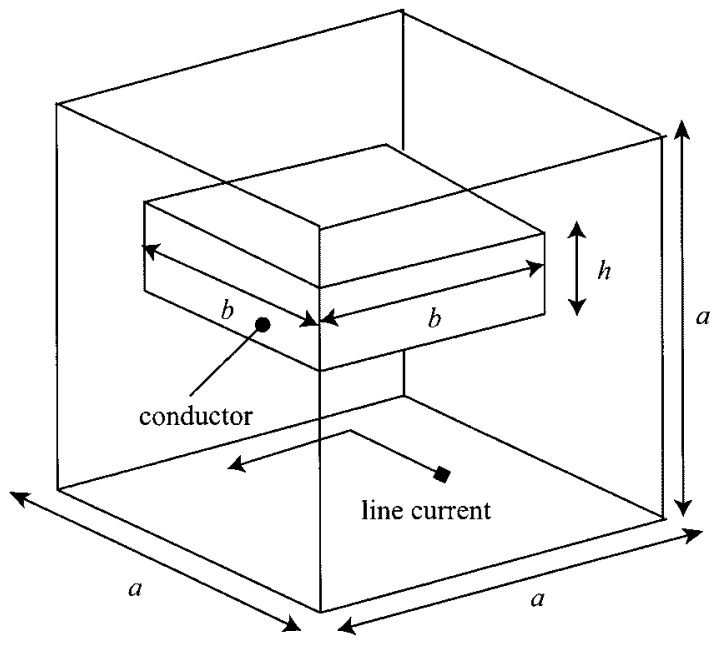

Fig. 1. Eddy-current problem (1/8) $\sigma=5.8 \times 10^{7}[\mathrm{~S} / \mathrm{m}], b=3 a / 4$, and $h=a / 4$.

TABLE I

FREQUENCY DEPENDENCE OF NUMBER OF ITERATIONS $N$ 1344 EDGES, 512 NODES, $a=20(\mathrm{~mm})$

\begin{tabular}{llrrrrr}
\hline & $f[\mathrm{~Hz}]$ & 100 & 50 & 10 & 1 & 0 \\
\hline & $\alpha$ & 18.3 & 9.15 & 4.56 & 2.29 & 0 \\
\hline & $\kappa$ & 472 & 651 & 4709 & 47092 & 35.5 \\
\hline CG & $\mathrm{A}$ & 111 & 121 & 151 & 150 & 38 \\
& $\mathrm{~A}-\mathrm{V}$ & 81 & 90 & 103 & 121 & 38 \\
\hline ICCG & $\mathrm{A}$ & 43 & 51 & 65 & 78 & $\mathbf{1 4}$ \\
& $\mathrm{A}-\mathrm{V}$ & 17 & 16 & 16 & 16 & $\mathbf{1 4}$ \\
\hline
\end{tabular}

convergence of ICCG for the A-V method is much better than that for the A method, and is almost the same as that for the static case over all frequencies. This suggests that the preconditioning plays a crucial role in the A-V method but not in the A method.

Fig. 2 shows the convergence process of the ICCG method. Table II summarizes the dependence of $N$ on the model size $a$. It can be seen here that the convergence depends on $a$ in a similar way as $f$ in Table $\mathrm{I}$.

\section{SPECtra of Finite-Element Matrix}

To study these phenomena further, we compute the singular-value distribution of the finite-element matrices $[K]$ as well as the preconditioned matrix $[P][K]$, where $[P]$ denotes the preconditioning matrix which is obtained by the incomplete Cholesky factorization of $[K]$. These computations are performed also for $\left[K^{\prime}\right]$ and $\left[P^{\prime}\right]\left[K^{\prime}\right]$.

The singular value $\sigma_{i}$ of a complex matrix $[A]$ satisfies $\sigma_{i}=$ $\sqrt{\mu_{i}},[A]^{*}[A]\left\{x_{i}\right\}=\mu_{i}\left\{x_{i}\right\}$, where $*$ denotes Hermitian conjugation and $\mu_{i}$ is the eigenvalue of $[A]^{*}[A]$. It is known that convergence of the CG method becomes better (worse) when the condition number, which would be defined by

$$
\kappa=\frac{\sigma_{\max }}{\sigma_{\min }^{0}}
$$

becomes smaller (larger) [7], [8], where $\sigma_{\max }$ and $\sigma_{\min }^{0}$ are the maximum singular value and minimum nonzero one of the matrix, respectively.

Fig. 3(a) and (b) show the distribution of the singular values of $[K]$ for A method and $\left[K^{\prime}\right]$ for A-V method, respectively. We

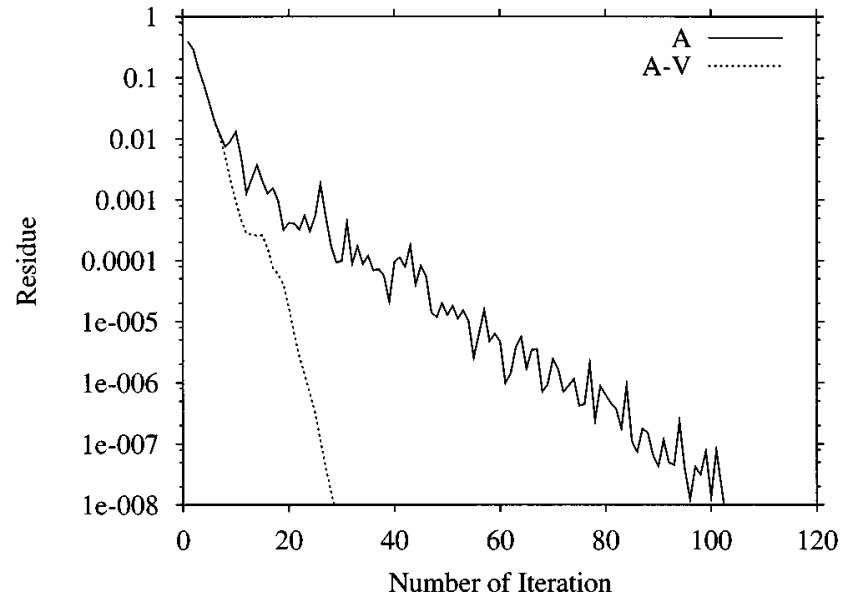

Fig. 2. Convergence process of ICCG $a=20(\mathrm{~mm}), f=50 \mathrm{~Hz}$.

TABLE II

SiZE DEPENDENCE OF NUMBER OF ITERATIONS $N$ $f=50[\mathrm{~Hz}],(\cdot, \cdot)$ DenOtes Numbers of EdGes AND Nodes

\begin{tabular}{llrrr|rrr}
\hline & & $(11520$, & $4096)$ & & $(1344$, & $512)$ & \\
\hline & $a[\mathrm{~mm}]$ & 40 & 20 & 10 & 40 & 20 & 10 \\
\hline & $\alpha$ & 36.3 & 9.00 & 2.27 & 36.3 & 9.00 & 2.27 \\
\hline CG & A & 206 & 227 & 257 & 93 & 121 & 154 \\
& A-V & 185 & 225 & 258 & 71 & 90 & 100 \\
\hline ICCG & A & 66 & 103 & 119 & 32 & 51 & 62 \\
& A-V & 31 & 29 & 29 & 18 & 16 & 16 \\
\hline
\end{tabular}

see that there are three groups in the spectra: the small singular values, the large singular values which form the long, horizontal belt and the singular values existing between the previous two groups. The small singular values in the first group represent the zero eigenvalues coming from the singularity of the finite-element matrices. Here, they are not exactly zero due to the numerical errors in the eigenvalue computation. Moreover, these zero singular values do not give any influence on the convergence [7].

Further numerical experiments reveal that the singular values in the last group are proportional to $\omega \sigma \mu$. On the other hand, the other two groups are not sensitive to it.

The singular values included in the last group are called the floating singular values for convenience. The condition number $\kappa$ becomes worse (larger) as $\omega \sigma \mu$ decreases. This deterioration of the condition number is caused by the floating singular values because their tail determines the minimum nonzero singular value $\sigma_{\min }^{0}$.

After preconditioning [see Fig. 3(c) and (d)], the floating singular values still exist in the A method, whereas they completely disappear in the $\mathrm{A}-\mathrm{V}$ method.

\section{THEORETICAL CONSIDERATION}

We consider the property of the differential equation of the quasi-static fields. In the metallic region, the differential equation for the A method is normalized to obtain

$$
\nabla^{\prime} \times\left(\nabla^{\prime} \times A\right)+\mathrm{j} \alpha \boldsymbol{A}=0 .
$$

When $\alpha$ is large, the second term of (8) is dominant while when $\alpha$ becomes small, the first term becomes dominant. For the 


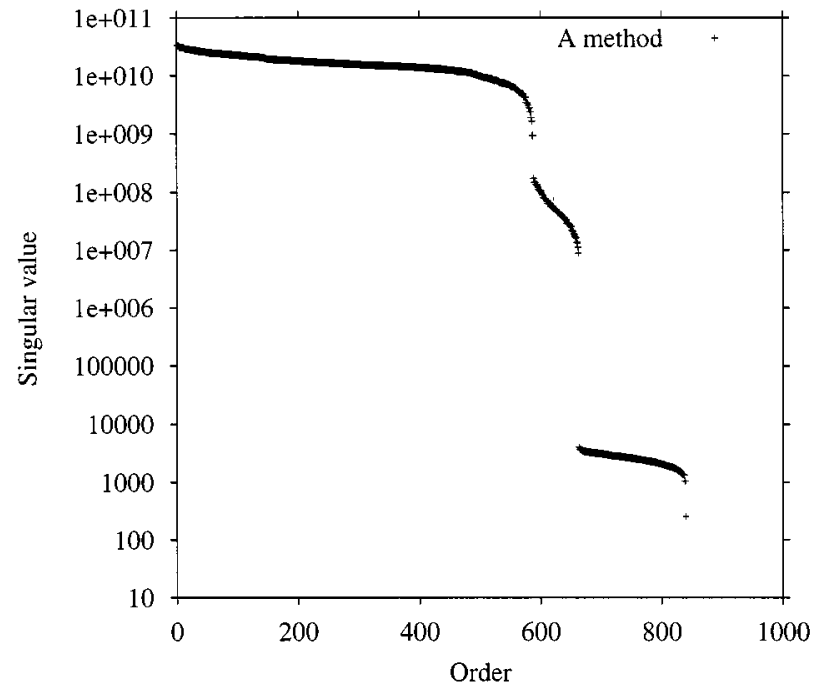

(a)

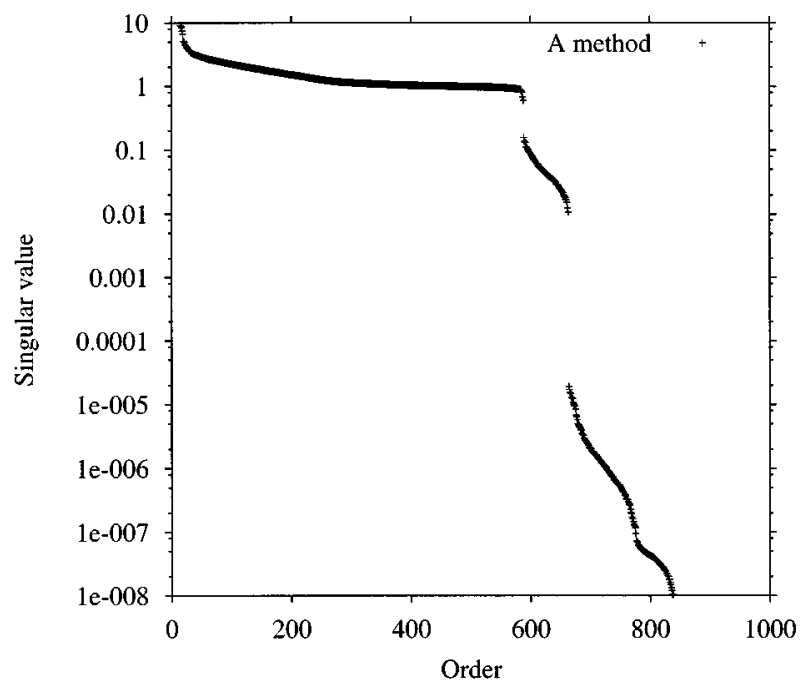

(c)

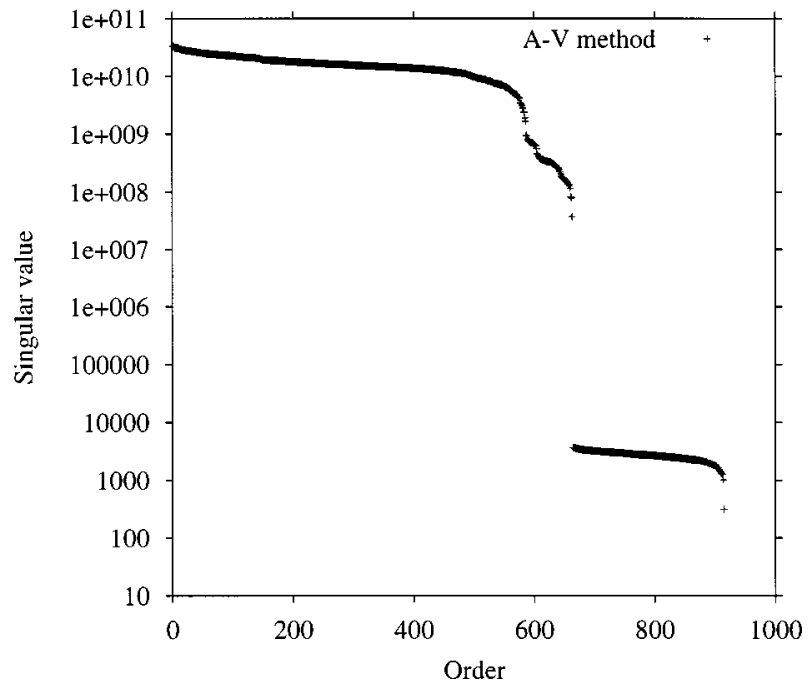

(b)

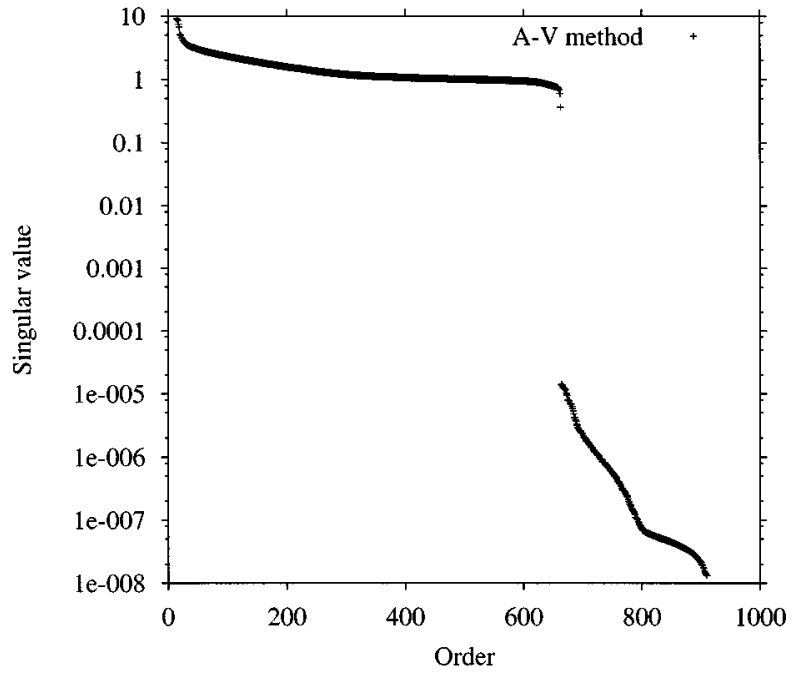

(d)

Fig. 3. Singular values of finite-element matrix $f=50 \mathrm{~Hz}, a=20 \mathrm{~mm}$. (a) A method (before preconditioning). (b) A-V method (before preconditioning). (c) A method (after preconditioning). (d) A-V method (after preconditioning).

above reason, $f$ and $a^{2}$ give the same influence on the equation as well as the convergence of the CG iteration.

Next we consider the property of the singular values. In the purely static cases at $f=0$, the matrices $\left[K_{0}\right]$ and $\left[K_{0}^{\prime}\right]$ have the zero eigenvalues, where the suffix 0 denotes the static situation. When frequency increases to a small value from zero, the zero eigenvalues would also increase to small values because $[K]$ and $\left[K^{\prime}\right]$ are the perturbed matrices of $\left[K_{0}\right]$ and $\left[K_{0}^{\prime}\right]$, respectively. Due to such small singular values, the condition number $\kappa$ becomes large, making the convergence of the CG method worse. Because the similar discussion can be made also for $[K]^{*}[K]$ and $\left[K^{\prime}\right]^{*}\left[K^{\prime}\right]$, the appearance of the floating singular value can be understood.

Now we consider the preconditioned matrix, written by $[P][K]$, of the A method. At the static case, the matrix $\left[P_{0}\right]\left[K_{0}\right]$, and also the Hermitian matrix $\left[K_{0}\right]^{*}\left[P_{0}\right]^{*}\left[P_{0}\right]\left[K_{0}\right]$ clearly have the zero eigenvalues.

There would be small changes in the eigenvalues of the matrices when frequency is increased to a small value from zero since $[P][K]$ can be regarded as the perturbed matrix of $\left[P_{0}\right]\left[K_{0}\right]$. Because this is also valid for $[K]^{*}[P]^{*}[P][K]$, the floating singular value remain after preconditioning.

On the other hand, in the case of the A-V method, the zero matrices in $\left[K_{0}^{\prime}\right]$ become nonzero ones when frequency increases from zero to small values. Due to such newly appeared nonzero matrices, the structure of $\left[P^{\prime}\right]\left[K^{\prime}\right]$ would be much different from that of $\left[P_{0}^{\prime}\right]\left[K_{0}^{\prime}\right]$. Therefore, although $\left[K^{\prime}\right]$ is regarded as the perturbed matrix of $\left[K_{0}^{\prime}\right]$, the preconditioned matrix $\left[P^{\prime}\right]\left[K^{\prime}\right]$ is no longer the perturbed matrix of $\left[P_{0}^{\prime}\right]\left[K_{0}^{\prime}\right]$. Consequently, the floating singular values are expected to disappear after preconditioning. The above hypothesis is tested for small matrices in the next section.

Finally, what happens in the A method is summarized as follows. When frequency, which can be replaced by $\alpha$, becomes smaller, then the nonzero minimum singular value $\sigma_{\min }^{0}$ becomes smaller, the condition number $\kappa$ larger, and thus the convergence worse. This is also valid in the $\mathrm{A}-\mathrm{V}$ method without preconditioning. Since the A-V has no floating singular values 
after preconditioning, the corresponding ICCG convergence is independent of $\alpha$. They are consistent with the numerical results shown in the previous section.

\section{Simple Test PRoblem}

Let us consider the matrix

$$
[k]=\left[k_{0}\right]+\epsilon[l]
$$

where

$$
\left[k_{0}\right]=\left[\begin{array}{ccc}
2 & 1 & 1 \\
1 & 1 & 0 \\
1 & 0 & 1
\end{array}\right]
$$

and $\epsilon$ is a small parameter. This matrix would possess the substantial property of the matrix $[K]$ for the A method, that is, the singular matrix $\left[k_{0}\right]$ is perturbed by the regular matrix $[I]$ with the small parameter $\epsilon$. The matrix

$$
\{g\}=\{1,-1,-1\}^{t}
$$

plays the role similar to the vector belonging to the kernel of $[C]^{t}[\nu][C]$.

The eigenvalues of $[k]$ are $\epsilon, 1+\epsilon$, and $3+\epsilon$. The corresponding eigenvectors are given by $\{g\},\{0,-1,1\}^{t}$ and $\{2,1,1\}^{t}$, respectively. The eigenvalue $\epsilon$ corresponds to the floating singular value which deteriorates the convergence of $\mathrm{CG}$ at low frequencies.

The matrix preconditioned by $[p]$ generated by the incomplete Cholesky factorization is

$$
\left[p_{0}\right]\left[k_{0}\right]=\left[\begin{array}{rrr}
1.0000 & 0.5000 & 0.5000 \\
0.0000 & 1.0000 & -1.0000 \\
0.0000 & -1.0000 & 1.0000
\end{array}\right]
$$

when $\epsilon=0$, and

$$
[p][k]=\left[\begin{array}{rrr}
1.0000 & 0.4983 & 0.4983 \\
0.0000 & 1.0000 & -0.9970 \\
0.0000 & -0.9970 & 1.0000
\end{array}\right]
$$

when $\epsilon$ is set to $1.0 \times 10^{-3}$. It can be seen that $\left[p_{0}\right]\left[k_{0}\right]$ is perturbed to obtain $[p][k]$. Thus, the floating singular values are expected to exist after preconditioning. In fact, the singular values of $[p][k]$ are $2.446 \times 10^{-3}, 1.223$, and 1.997 when $\epsilon=$ $1.0 \times 10^{-3}$, and the first one, which changes proportional to $\epsilon$, corresponds to the floating singular value.

The matrix corresponding to $\left[K^{\prime}\right]$ for the $\mathrm{A}-\mathrm{V}$ method is defined by

$$
\begin{aligned}
{\left[k^{\prime}\right] } & =\left[\begin{array}{cc}
{\left[k_{0}\right]+\epsilon[I]} & \epsilon\{g\} \\
\epsilon\{g\}^{t} & \epsilon\{g\}^{t}\{g\}
\end{array}\right] \\
& =\left[\begin{array}{cccc}
2+\epsilon & 1 & 1 & \epsilon \\
1 & 1+\epsilon & 0 & -\epsilon \\
1 & 0 & 1+\epsilon & -\epsilon \\
\epsilon & -\epsilon & -\epsilon & 3 \epsilon
\end{array}\right] .
\end{aligned}
$$

The eigenvalues of $\left[k^{\prime}\right]$ are $0,4 \epsilon, 1+\epsilon$, and $3+\epsilon$, and the corresponding eigenvectors are given by $\left\{g^{t},-1\right\}^{t}$, $\{1,-1,-1,3\}^{t},\{0,-1,1,0\}^{t}$ and $\{2,1,1,0\}^{t}$, respectively.

The incomplete Cholesky factorization of $\left[k^{\prime}\right]$ with the preconditioning matrix $\left[p^{\prime}\right]$ results in

$$
\left[p_{0}^{\prime}\right]\left[k_{0}^{\prime}\right]=\left[\begin{array}{rrrr}
1.0000 & 0.5000 & 0.5000 & 0.0000 \\
0.0000 & 1.0000 & -1.0000 & 0.0000 \\
0.0000 & -1.0000 & 1.0000 & 0.0000 \\
0.0000 & 0.0000 & 0.0000 & 0.0000
\end{array}\right]
$$

when $\epsilon=0$, and

$$
\left[p^{\prime}\right]\left[k^{\prime}\right]=\left[\begin{array}{rrrr}
1.0000 & 0.5000 & 0.5000 & 0.0000 \\
0.0000 & 0.9985 & -0.9985 & 0.0000 \\
0.0000 & -0.9985 & 0.9985 & 0.0000 \\
0.0000 & -0.5000 & -0.5000 & 1.0000
\end{array}\right]
$$

when $\epsilon$ is set to $1.0 \times 10^{-3}$.

The matrix $\left[p^{\prime}\right]\left[k^{\prime}\right]$ can no longer be regarded as the perturbed matrix of $\left[p_{0}^{\prime}\right]\left[k_{0}^{\prime}\right]$. The floating singular values are, therefore, expected to disappear now. In fact, the singular values of $\left[p^{\prime}\right]\left[k^{\prime}\right]$ are $0,1.000,1.414$, and 1.997 when $\epsilon=1.0 \times 10^{-3}$. There exist no floating singular values.

\section{CONCLUSION}

The convergence of the CG iteration becomes worse when $\alpha$ decreases. This phenomenon is caused by the decrease in the floating singular values. The preconditioning eliminates such floating singular values in the $\mathrm{A}-\mathrm{V}$ method while not in the A method. This explains the better convergence of the A-V method.

\section{ACKNOWLEDGMENT}

The authors would like to thank A. Kameari, M. Hano, and A. Bossavit for helpful discussions.

\section{REFERENCES}

[1] A. Kameari, "Three dimensional eddy current calculation using edge elements for magnetic vector potential," in Appl. Electromagnetics in Materials. $\quad$ New York: Pergamon, 1986, pp. 225-236.

[2] O. Biro and K. Preis, "On the use of the magnetic vector potential in the finite element analysis of three-dimensional eddy currents," IEEE Trans. Magn., vol. 25, pp. 3145-3159, 1989.

[3] K. Fujiwara, T. Nakata, and H. Ohashi, "Improvement of convergence characteristics of ICCG method for the $A-\varphi$ method using edge elements," IEEE Trans. Magn., vol. 32, pp. 804-807, 1996.

[4] A. Kameari and M. Hano, private communication.

[5] A. Bossavit, Computational Electromagnetism. New York: Academic, 1998.

[6] M. Hano, "Eddy current analysis by using high-order Nedelec's elements" (in Japanese), in Proc. Japanese IEE Conf., SAO0-10, 2000, pp. 55-60.

[7] H. Igarashi, "On the property of the curl-curl matrix in finite element analysis with edge elements," IEEE Trans. Magn., vol. 37, pp. 3129-3132, 2001.

[8] S. Kaniel, "Estimates for some computational techniques in linear algebra,” Math. Comput., pp. 369-378, 1966. 